\title{
Clock Speed
}

National Cancer Institute

\section{Source}

National Cancer Institute. Clock Speed. NCI Thesaurus. Code C63627.

The fundamental rate in cycles per second at which a computer performs its most basic operations such as adding two numbers or transfering a value from one register to another. 